\title{
Les sciences spatiales
}

\section{Adapter la recherche française aux enjeux de l'Espace}

RAPPORT SUR LA SCIENCE ET LA TECHNOLOGIE N³0

Animateur : Jean-Loup Puget

ACADÉMIE DES SCIENCES

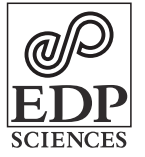

17, avenue du Hoggar

Parc d'activités de Courtabœuf, BP 112

91944 Les Ulis Cedex A, France 
Rapports sur la science et la technologie

- Sciences et pays en développement. Afrique subsaharienne francophone RST n 21, 2006.

- La recherche spatiale française RST n²2, 2006.

- L'épidémiologie humaine. Conditions de son développement en France, et rôle des mathématiques

RST n²3, 2006.

- La maîtrise des maladies infectieuses. Un défi de santé publique, une ambition médico-scientifique

RST n² 24, 2006.

- Les eaux continentales

RST $n^{\circ} 25,2006$.

- La fusion nucléaire : de la recherche fondamentale à la production d'énergie?

RST n²6, 2006.

- Cycles biogéochimiques et écosystèmes continentaux RST n²7, 2007.

- Hormones, santé publique et environnement RST n²8, 2008.

- Événements climatiques extrêmes. Réduire les vulnérabilités des systèmes écologiques et sociaux

RST n²9, 2010.

\section{Imprimé en France}

(c) 2010, EDP Sciences, 17, avenue du Hoggar, BP 112, Parc d'activités de Courtabœuf, 91944 Les Ulis Cedex A

Tous droits de traduction, d'adaptation et de reproduction par tous procédés réservés pour tous pays. Toute reproduction ou représentation intégrale ou partielle, par quelque procédé que ce soit, des pages publiées dans le présent ouvrage, faite sans l'autorisation de l'éditeur est illicite et constitue une contrefaçon. Seules sont autorisées, d'une part, les reproductions strictement réservées à l'usage privé du copiste et non destinées à une utilisation collective, et d'autre part, les courtes citations justifiées par le caractère scientifique ou d'information de l'œuvre dans laquelle elles sont incorporées (art. L. 122-4, L. 122-5 et L. 335-2 du Code de la propriété intellectuelle). Des photocopies payantes peuvent être réalisées avec l'accord de l'éditeur. S'adresser au : Centre français d'exploitation du droit de copie, 3, rue Hautefeuille, 75006 Paris. Tél. : 0143269535. 


\section{Académie des sciences}

\section{Rapport Science ef Technologie}

Le Comité interministériel du 15 juillet 1998, à l'initiative du ministre de l'Éducation nationale, de la Recherche et de la Technologie, a confié à l'Académie des sciences l'établissement du rapport biennal sur l'état de la science et de la technologie.

Pour répondre à cette demande, l'Académie des sciences a mis en place en son sein le Comité "Rapport Science et Technologie » (RST), chargé de choisir les sujets d'étude et de suivre les travaux.

Chaque thème retenu est conduit par un groupe de travail animé par un membre ou un correspondant de l'Académie, entouré d'experts.

Chaque rapport est soumis au Comité RST, à un Groupe de lecture critique, et à l'Académie des sciences.

Depuis 1999, vingt-neuf rapports ont ainsi été édités et remis au ministre chargé de la Recherche. 
This page intentionally left blank 